\title{
Asymptotic inversion of the Erlang B formula
}

\author{
J.S.H. van Leeuwaarden ${ }^{\circ} \quad \& \quad$ N.M. Temme $e^{\bullet}$
}

October 31,2008

\begin{abstract}
The Erlang B formula represents the steady-state blocking probability in the Erlang loss model or $M / M / s / s$ queue. We derive asymptotic expansions for the offered load that matches, for a given number of servers, a certain blocking probability. In addressing this inversion problem we make use of various asymptotic expansions for the incomplete gamma function. A similar inversion problem is investigated for the Erlang $\mathrm{C}$ formula.
\end{abstract}

2000 Mathematics Subject Classification: 60K25, 33B20, 41A60.

Keywords \& Phrases: Erlang B formula, Erlang C formula, Erlang loss model, queueing theory, incomplete gamma function, asymptotic inversion, asymptotic analysis.

\section{Introduction}

The Erlang B formula is perhaps the most classical result in queueing theory, representing the steady-state blocking probability in the Erlang loss model or $M / M / s / s$ queue. Derived by A.K. Erlang in 1917, it has been the subject of extensive study ever since. Its wide range of applications made many researchers investigate the Erlang B formula from various angles. This paper deals with the open problem of inverting the Erlang B formula.

The reader is referred to the elucidation of Erlang's work and era in Brockmeyer et al. [2], and to Cooper [3], Kosten [11], Riordan [12], Syski [13] and Whitt [18] for some more of the historical flavor. The Erlang loss model has $s$ homogeneous servers working in parallel and no extra waiting space. Customers that find all $s$ servers busy upon arrival are blocked (lost). It is further assumed that customers arrive according to a Poisson process with rate $\nu$ and that the service times are independent and exponentially distributed with mean $1 / \mu$. We define the offered load as $\lambda=\nu / \mu$, and the service utilization as $\rho=\lambda / s$. The Erlang B formula is then given by

$$
B(s, \lambda)=\frac{\lambda^{s} / s !}{\sum_{k=0}^{s} \lambda^{k} / k !}
$$

The work of Jagerman [8] contains a large variety of exact, asymptotic and approximative representations of $B(s, \lambda)$, and serves as a standard reference. As a desirable further investigation, Jagerman mentions in the conclusion of [8] the inversion of $B(s, \lambda)$, which boils down to finding the load $\lambda$ such that

$$
B(s, \lambda)=p,
$$

\footnotetext{
${ }^{\circ}$ Eindhoven University of Technology and EURANDOM, P.O. Box 513, 5600 MB Eindhoven, The Netherlands. Email address: j.s.h.v.leeuwaarden@tue.nl

• CWI, P.O. Box 94079, 1090 GB Amsterdam, The Netherlands. Email address: nico.temme@cwi.nl
} 
for some $p \in(0,1)$ and $s \in \mathbb{N}$. A few years later, Jagerman [9] himself developed an efficient numerical algorithm based on Newton's method. For the strongly related problem of solving (1.2) for $s$ given $\lambda$ and $p$, Jagerman [9] also proposed Newton's method, and bounds on $s$ were derived in [1] and [6]. An exact and comprehensive treatment of the inversion problem, though, seems still largely missing from the literature.

As pointed out by Jagerman and many others, the Erlang B formula can be expressed in terms of the incomplete gamma function. Temme [15] has considered the inversion problem for the incomplete gamma function based on a uniform asymptotic expansion developed in [14]. We shall derive various asymptotic expansions for the inverse $\lambda$ of the Erlang B formula using asymptotic expansions for the incomplete gamma function. We also show how these asymptotic techniques can be transferred to a similar inversion problem for the Erlang $\mathrm{C}$ formula, which represents the steady-state probability of delay in the Erlang delay model or $M / M / s$ queue. That the same machinery can be applied is not surprising, since the Erlang B and C formulae are intrinsically intertwined, cf. (7.1).

Of fundamental importance in the performance analysis of stochastic systems, the Erlang $\mathrm{B}$ and $\mathrm{C}$ formulae have found numerous applications. The inverse is of concern in dimensioning problems. The results in this paper may contribute to future investigations in two ways.

First, we derive asymptotic expansions of which the first few terms serve as highly accurate approximations. Such approximations may reduce computation time in large optimization problems or simulation settings, or may allow for an exact, formal, solution of an optimization problem in which the true inverse is replaced by its approximation. Moreover, the second terms in the asymptotic expansions render both quantitative and qualitative insight into the speed of convergence to the asymptotic regime at hand.

Second, while the first few terms of the expansion yield sharp approximations already, even sharper results may be obtained by either including more terms, or using Newton's method; see (6.1) and (7.16). The starting value of Newton's method, depending on $p$, follows from our asymptotic framework. This avoids unfavorable situations like large errors in successive approximations and, even worse, approximations that are found outside the definition range of the variable to be computed; see [5, Chapter 10] for more examples. Then, Newton's method, with at most 4 iterations, yields the inverse up to at least 10 digits precision, irrespective of $s$ and $p$.

\section{Outline}

The main objective of this paper is to find the $\lambda$-value of Equation (1.2) for a large value of $s$, where $B(s, \lambda)$ is the Erlang B formula defined in (1.1). Throughout, we shall use the representation

$$
B(s, \lambda)^{-1}=1+s ! e^{\lambda} \lambda^{-s} Q(s, \lambda),
$$

with $Q(s, \lambda)$ the incomplete gamma function given by

$$
Q(s, \lambda)=\frac{\Gamma(s, \lambda)}{\Gamma(s)}=\frac{1}{\Gamma(s)} \int_{\lambda}^{\infty} t^{s-1} e^{-t} d t .
$$

Clearly, (2.1) has meaning for non-integral $s$. Fig. 1 shows graphs of $B(s, \rho s)$ for $0 \leq$ $\rho \leq 5$ and $s=1,3,5,10,25,50,100,1000$ (the far left curve is for $s=1$, the far right one for $s=1000$ ). These graphs suggest the consideration of three regimes corresponding to small, medium and large blocking probability. The transition from small to medium 


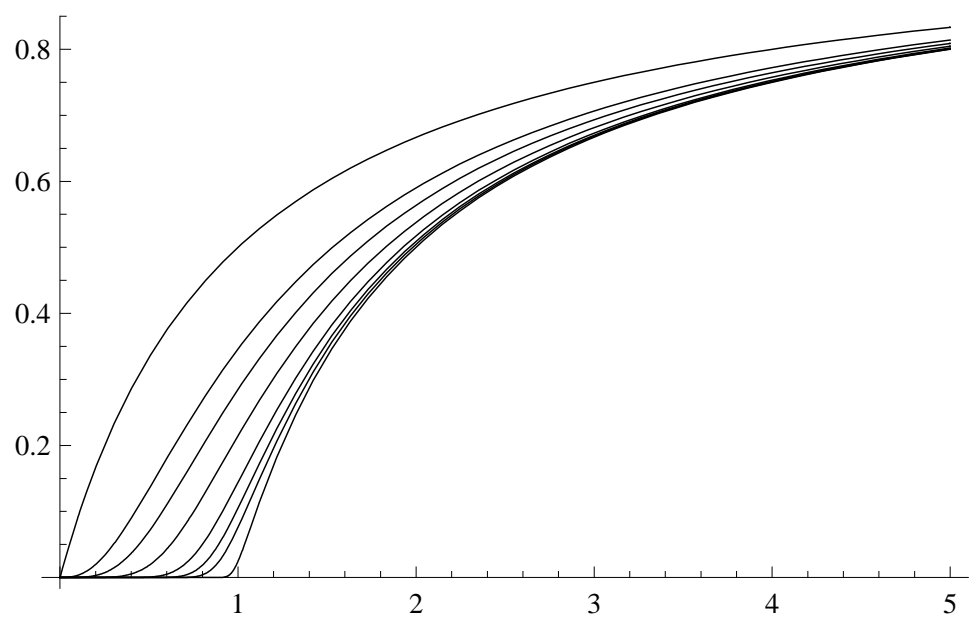

Figure 1: Graphs of $B(s, \rho s)$ for $0 \leq \rho \leq 5$ and $s=1,3,5,10,25,50,100,1000$ (the far left curve is for $s=1$, the far right one for $s=1000$.

$p$, as $\lambda$ increases, can occur rather abruptly. A small $p$ typically corresponds to $\lambda<s$, while a large $p$ corresponds to $\lambda>s$. To further investigate this relation, we derive from asymptotic properties of $Q(s, \lambda)$, see $\S 5$ or [8, p. 540],

$$
B(s, s) \sim \frac{6}{4+3 \sqrt{2 \pi s}}, \quad s \rightarrow \infty,
$$

and the relative error of this estimate is less than one percent for $s>5$. When $p$ in (1.2) is less (larger) than the right-hand side of (2.3), we have $\lambda\langle s(\lambda>s)$, approximately.

We shall derive various asymptotic expansions for the inverse $\lambda$ of (1.2), using existing asymptotic expansions for the incomplete gamma function. Based on the above observations, we shall consider three asymptotic regimes:

Large blocking probability. This typically corresponds to an overload situation $\lambda \gg s$, in which case the Erlang B formula is well approximated by $B(s, \lambda) \approx(\lambda-s) / \lambda$ and hence, for given $s$ and $p, \lambda \approx s /(1-p)$. In $\S 3$ we derive the higher terms of the expansion for $\lambda$ using two standard asymptotic expansions for the incomplete gamma function.

Small blocking probability. This corresponds to the underload situation $\lambda<s$, in which case the Erlang B formula is well approximated by $B(s, \lambda) \approx e^{-\lambda} \lambda^{s} / s !$, leading to a first order approximation for the inverse $\lambda \sim \rho_{0} s$ for $s \rightarrow \infty$, where $\rho=\rho_{0}$ solves

$$
\left(p e^{s} s ! s^{s}\right)^{1 / s}=\rho e^{1-\rho} .
$$

In $\S 4$ we identify some of the higher terms of the asymptotic expansion $\lambda \sim \rho_{0} s+\rho_{1}+$ $\rho_{2} s^{-1}+\ldots$ using the connection between $Q(s, \lambda)$ and the confluent hypergeometric function. All higher terms can be expressed in terms of $\rho_{0}$.

General case. For all other cases with $p \in(0,1)$ we employ a uniform asymptotic expansion for the incomplete gamma function derived in [14], in which the standard normal distribution function (or error function) is the leading approximant. In [15] the first coefficients in the expansion were derived by using a perturbation method for a differential equation, but this approach cannot be transferred directly to the inversion of the Erlang B formula. We describe an alternative method that uses Taylor expansions and the connection between the derivatives of $Q(s, \lambda)$ and Hermite polynomials. This is done in $\S 5$. 
Table 1: Numerical results of the approximation (3.4) for $p=\frac{4}{5}\left(q=\frac{1}{4}\right)$ and several values of $s$.

\begin{tabular}{rccc}
$s$ & $\lambda$ & $B(s, \lambda)$ & rel. error \\
\hline 5 & $2.38910^{1}$ & $8.00610^{-1}$ & $7.5310^{-4}$ \\
10 & $4.89610^{1}$ & $8.00710^{-1}$ & $8.4510^{-4}$ \\
25 & $1.24210^{2}$ & $8.00710^{-1}$ & $9.1910^{-4}$ \\
50 & $2.49710^{2}$ & $8.00810^{-1}$ & $9.4710^{-4}$ \\
75 & $3.75210^{2}$ & $8.00810^{-1}$ & $9.5710^{-4}$ \\
100 & $5.00710^{2}$ & $8.00810^{-1}$ & $9.6210^{-4}$ \\
250 & $1.25410^{3}$ & $8.00810^{-1}$ & $9.7110^{-4}$ \\
500 & $2.50910^{3}$ & $8.00810^{-1}$ & $9.7410^{-4}$ \\
1000 & $5.01810^{3}$ & $8.00810^{-1}$ & $9.7510^{-4}$
\end{tabular}

In $\S 6$ we discuss Newton's method for further improving the accuracy of the approximation for the inverse found by our asymptotic techniques, and finally, in $\S 7$, we apply the same asymptotic techniques to address the inverse problem for the Erlang $\mathrm{C}$ formula.

\section{Inversion for $p \uparrow 1$}

When $p \uparrow 1$ the solution $\lambda$ of (1.2) satisfies $\lambda \gg s$ (see $\S 2$ ), and we can use the standard asymptotic expansion of the incomplete gamma function (see [17, p. 280])

$$
\Gamma(s, \lambda) \sim \lambda^{s-1} e^{-\lambda} \sum_{n=0}^{\infty} \frac{(-1)^{n}(1-s)_{n}}{\lambda^{n}},
$$

where $(\alpha)_{n}$ is the Pochhammer symbol defined by $(\alpha)_{0}=1$ and $(\alpha)_{n}=\Gamma(\alpha+n) / \Gamma(\alpha)$ for $n=1,2,3, \ldots$. When $\alpha$ is a non-positive integer we use the following interpretation of the Pochhammer symbol. We have for $m, n=0,1,2, \ldots$

$$
(-m)_{n}= \begin{cases}0, & \text { if } n>m, \\ (-1)^{n} m ! /(m-n) !, & \text { if } n \leq m\end{cases}
$$

It follows that for $s=1,2,3, \ldots$ the series in (3.1) terminates and contains $s$ terms, giving an exact representation.

By using (2.2) and the asymptotic expansion, the asymptotic inversion problem reads

$$
q=\frac{1-p}{p} \sim \frac{s}{\lambda} \sum_{n=0}^{\infty} \frac{(-1)^{n}(1-s)_{n}}{\lambda^{n}} .
$$

When $q$ is small we can find an asymptotic expansion of the solution $\lambda$ in terms of a series in powers of $q$, which holds whether or not $s$ is large. By inverting (3.3) we find

$$
\lambda^{-1} \sim \frac{q}{s}-\frac{(s-1) q^{2}}{s^{2}}+\frac{(s-1) q^{3}}{s^{2}}-\frac{(s-1)\left(s^{2}+1\right) q^{4}}{s^{4}}+\ldots,
$$

and we see that it gives the exact result $\lambda^{-1}=q$ when $s=1$. 
Table 2: Numerical results of the approximation (3.4) for $s=100$ and several values of $p$.

\begin{tabular}{cccc}
$p$ & $\lambda$ & $B(s, \lambda)$ & rel. error \\
\hline 0.60 & $3.08210^{2}$ & $6.771110^{-1}$ & $1.2910^{-01}$ \\
0.70 & $3.43310^{2}$ & $7.099210^{-1}$ & $1.4210^{-02}$ \\
0.80 & $5.00710^{2}$ & $8.007710^{-1}$ & $9.6210^{-04}$ \\
0.90 & $9.99010^{2}$ & $9.000210^{-1}$ & $1.6710^{-05}$ \\
0.99 & $9.99910^{3}$ & $9.900010^{-1}$ & $1.0410^{-10}$
\end{tabular}

In Table 1 we give the results of numerical computations. We take $p=\frac{4}{5}\left(q=\frac{1}{4}\right)$ and several values of $s$. We have used the terms in (3.4) up to (and including) the term with $q^{4}$, as shown in (3.4). We see a rather uniform error for the used values of $s$. The relative error is $|B(s, \lambda) / p-1|$. In Table 2 we give the results of the approximation (3.4) for $s=100$ and several values of $p$.

\subsection{Using an alternative asymptotic expansion}

Next we try to solve (1.2) by using the asymptotic expansion of $\Gamma(s, \lambda)$ given in Appendix A; see (A.6). It follows that we can write (1.2) in the form of the asymptotic identity

$$
q=r \sum_{n=0}^{\infty} \frac{G_{n}(r)}{\lambda^{n}}, \quad r=\frac{s}{\lambda} .
$$

Because $G_{0}(r)=1 /(1-r)$, the first term approximation gives

$$
q=\frac{r}{1-r} \quad \Rightarrow \quad r=\frac{q}{1+q} \quad \Rightarrow \quad \lambda=\frac{s(1+q)}{q},
$$

compare with (3.4). Note that this first term follows from the approximation $B(s, \lambda)^{-1} \approx$ $\frac{\lambda}{\lambda-s}$ for $\lambda>s$. In [9], Equation (46), it is proved that in fact $B(s, \lambda)^{-1} \leq \frac{\lambda}{\lambda-s}$. To find higher approximations we write

$$
\lambda_{0}=\frac{s(1+q)}{q}
$$

which gives $q=s /\left(\lambda_{0}-s\right)$, and Equation (3.5) can be written as

$$
\frac{s}{\lambda_{0}-s}=r \sum_{n=0}^{\infty} \frac{G_{n}(r)}{\lambda^{n}}, \quad r=\frac{s}{\lambda} .
$$

In this section we have $p \uparrow 1$, hence $q \downarrow 0$, and we assume that $\lambda_{0}$ is large. Thus, we try to find a solution $\lambda^{-1}$ of (3.8) of the form

$$
\lambda^{-1} \sim \lambda_{1} \lambda_{0}^{-1}+\lambda_{2} \lambda_{0}^{-2}+\lambda_{3} \lambda_{0}^{-3}+\lambda_{4} \lambda_{0}^{-4}+\lambda_{5} \lambda_{0}^{-5}+\ldots,
$$

and we find by series manipulations

$$
\lambda_{1}=\lambda_{2}=1, \quad \lambda_{3}=s, \quad \lambda_{4}=1-s+s^{2}, \quad \lambda_{5}=-4+7 s-3 s^{2}+s^{3} .
$$

In Table 3 we give the results of numerical computations. We take $p=\frac{4}{5}\left(q=\frac{1}{4}\right)$ and several values of $s$. We have used in (3.9) the terms up to (and including) the term with 
Table 3: Numerical results of the approximation (3.9) for $p=\frac{4}{5}\left(q=\frac{1}{4}\right)$ and several values of $s$.

\begin{tabular}{rccc}
$s$ & $\lambda$ & $B(s, \lambda)$ & rel. error \\
\hline 5 & $2.38244110^{1}$ & $8.00044910^{-1}$ & $5.6210^{-5}$ \\
10 & $4.87934410^{1}$ & $8.00027710^{-1}$ & $3.4710^{-5}$ \\
25 & $1.23773710^{2}$ & $8.00013610^{-1}$ & $1.7010^{-5}$ \\
50 & $2.48766910^{2}$ & $8.00007310^{-1}$ & $9.1910^{-6}$ \\
75 & $3.73764610^{2}$ & $8.00005010^{-1}$ & $6.3010^{-6}$ \\
100 & $4.98763510^{2}$ & $8.00003810^{-1}$ & $4.7910^{-6}$ \\
250 & $1.24876110^{3}$ & $8.00001610^{-1}$ & $1.9710^{-6}$ \\
500 & $2.49876110^{3}$ & $8.00000810^{-1}$ & $9.9110^{-7}$ \\
1000 & $4.99876010^{3}$ & $8.00000410^{-1}$ & $4.9810^{-7}$
\end{tabular}

Table 4: Numerical results of the approximation (3.9) for $s=100$ and several values of $p$.

\begin{tabular}{cccc}
$p$ & $\lambda$ & $B(s, \lambda)$ & rel. error \\
\hline 0.60 & $2.48451210^{2}$ & $6.00159210^{-1}$ & $2.6510^{-04}$ \\
0.70 & $3.31950010^{2}$ & $7.00032910^{-1}$ & $4.7010^{-05}$ \\
0.80 & $4.98763510^{2}$ & $8.00003810^{-1}$ & $4.7910^{-06}$ \\
0.90 & $9.98891310^{2}$ & $9.00000110^{-1}$ & $1.1910^{-07}$ \\
0.99 & $9.99899010^{3}$ & $9.90000010^{-1}$ & $9.9010^{-13}$
\end{tabular}

$\lambda_{0}^{-4}$. The relative error is $|B(s, \lambda) / p-1|$. We see a better performance compared with the results in Table 1. In Table 4 we give the results of the approximation (3.9) for fixed $s=100$ and several values of $p$. Again, we see a better performance compared with the results in Table 2.

\section{Inversion for $p \downarrow 0$}

Another relatively simple asymptotic inversion is possible when $p$ is small. In that case we expect a solution of (1.2) for $\lambda<s$ (see $\S 2$ ). We use the relation

$$
\Gamma(s, \lambda)=\Gamma(s)-\gamma(s, \lambda)
$$

and the convergent expansion (see [17, p. 279] or Theorem 5 in Jagerman [8])

$$
\gamma(s, \lambda)=\lambda^{s} e^{-\lambda} \sum_{n=0}^{\infty} \frac{\lambda^{n}}{(s)_{n+1}},
$$

which can also be viewed as an asymptotic expansion for large values of $s$, with $\lambda$ fixed. For the asymptotic inversion, however, it seems better to use the asymptotic expansion given in Appendix B, see (B.6). When we use this in (2.1) we have the asymptotic inversion problem

$$
\frac{1}{p}=1+s ! \lambda^{-s} e^{\lambda}-\sum_{n=0}^{\infty} \frac{g_{n}(\rho)}{s^{n}}, \quad \rho=\frac{\lambda}{s} .
$$


The expansion holds for large values of $s$, uniformly for fixed $\rho \in(0,1)$.

When $\lambda$ is small compared with $s$, the first term in the right-hand side of (4.1) is much larger than the second term. Hence, first we consider the inversion of

$$
\frac{1}{p}=s ! \lambda^{-s} e^{\lambda} .
$$

We write

$$
s !=\Gamma(s+1)=\sqrt{2 \pi s} e^{-s} s^{s} \Gamma^{*}(s),
$$

with, according to Stirling's formula,

$$
\Gamma^{*}(s)=1+\mathcal{O}\left(s^{-1}\right), \quad s \rightarrow \infty .
$$

Equation (4.4) can thus be written as

$$
\left(p \sqrt{2 \pi s} \Gamma^{*}(s)\right)^{1 / s}=\rho e^{1-\rho} .
$$

When $p$ is small we consider the solution $\rho$ of this equation in the interval $(0,1)$ and denote it by $\rho_{0}$. Observe that the right-hand side of (4.7) has a maximum at $\rho=1$ (that is, when $\lambda=s$ ), and a real solution is only possible when $p$ is small enough: $p$ should satisfy

$$
p \leq \frac{1}{\sqrt{2 \pi s} \Gamma^{*}(s)} .
$$

It follows that the method of this section fails if the equation in (4.7) does not have a real solution. In that case it is better to use a different method for the transition area $\lambda \sim s$ (see $\S 5$ ). In Fig. 4 the lower curve indicates where in (4.8) the equal sign holds. For pairs $(s, p)$ properly below this curve the method of this section can be used.

To solve equation (4.7) and to obtain higher approximations it is convenient to introduce the quantity $\eta$ defined by

$$
\frac{1}{2} \eta^{2}=\rho-1-\ln \rho, \quad \rho>0, \quad \operatorname{sign}(\eta)=\operatorname{sign}(\rho-1) .
$$

This relation plays a role in later sections also, and we give details on the inversion (that is, finding $\rho$ when $\eta$ is given) in Appendix $\mathrm{C}$.

It follows from (4.9) that

$$
e^{-s} s^{s} e^{\lambda} \lambda^{-s}=e^{\frac{1}{2} s \eta^{2}}
$$

and that we can write (4.4) in the form

$$
p \sqrt{2 \pi s} \Gamma^{*}(s)=e^{-\frac{1}{2} s \eta^{2}} .
$$

We denote the solution of this equation by $\eta_{0}$. Since we assume that $p$ satisfies (4.8) and that the corresponding $\rho$-value belongs to $(0,1]$, we have $\eta_{0} \leq 0$, that is,

$$
\eta_{0}=-\sqrt{-\frac{2}{s} \ln \left(p \sqrt{2 \pi s} \Gamma^{*}(s)\right)} .
$$

The corresponding value of $\rho$ follows from inverting the relation in (4.9) for $\rho \leq 1$, and is denoted by $\rho_{0}$. 
We next turn to the inversion of (4.3). By using (4.5) and (4.10), this equation can be written in the form

$$
\frac{1}{p}=1+\sqrt{2 \pi s} \Gamma^{*}(s) e^{\frac{1}{2} s \eta^{2}}-\sum_{n=0}^{\infty} \frac{g_{n}(\rho)}{s^{n}} .
$$

We eliminate $\sqrt{2 \pi s} \Gamma^{*}(s)$ by using (4.11) with $\eta=\eta_{0}$ and obtain

$$
p \sum_{n=0}^{\infty} \frac{g_{n}(\rho)}{s^{n}}=p-1+e^{\frac{1}{2} s\left(\eta^{2}-\eta_{0}^{2}\right)} .
$$

We assume that $\left|\eta-\eta_{0}\right|$ is small, and expand $\rho$ as

$$
\rho=\rho_{0}+\sum_{n=1}^{\infty} \rho_{n}\left(\eta-\eta_{0}\right)^{n},
$$

where the first few coefficients are given by

$$
\begin{aligned}
& \rho_{1}=-\frac{\eta_{0} \rho_{0}}{1-\rho_{0}}, \quad \rho_{2}=\frac{\rho_{0}\left(\eta_{0}^{2}-\left(1-\rho_{0}\right)^{2}\right)}{2\left(1-\rho_{0}\right)^{3}}, \\
& \rho_{3}=-\frac{\rho_{0} \eta_{0}\left(\eta_{0}^{2}\left(1+2 \rho_{0}\right)-3\left(1-\rho_{0}\right)^{2}\right)}{6\left(1-\rho_{0}\right)^{3}} .
\end{aligned}
$$

These values reduce to the first coefficients given in (C.4) when $\eta_{0} \rightarrow 0$. However, when $\eta_{0}=0$ we have $\rho_{0}=1$, and the given values $\rho_{j}$ cannot be computed straightforwardly. In fact, we need a limiting process for $\eta_{0} \rightarrow 0$, or a series expansion for small values of $\eta_{0}$. For example, by using (C.4) with $\eta$ and $\rho$ replaced with $\eta_{0}$ and $\rho_{0}$, respectively, we have

$$
\rho_{1}=1+\frac{2}{3} \eta_{0}+\mathcal{O}\left(\eta_{0}^{2}\right), \quad \rho_{2}=\frac{1}{3}+\frac{1}{12} \eta_{0}+\mathcal{O}\left(\eta_{0}^{2}\right) .
$$

In this section we assume that $\rho_{0}$ is strictly less than unity, and we don't need these expansions.

It turns out, after formal asymptotic series operations, that $\eta$ can be expanded in the form

$$
\eta=\eta_{0}+\eta_{1} s^{-1}+\eta_{2} s^{-2}+\ldots .
$$

After substituting (4.15) and (4.18) into (4.14), and comparing equal powers of $s$, we find

$$
\eta_{1}=\frac{1}{\eta_{0}} \ln \left(1+\frac{p \rho_{0}}{1-\rho_{0}}\right),
$$

and

$$
\eta_{2}=-\frac{\eta_{1}^{2}\left(1-\rho_{0}\right)^{2}\left(1-\rho_{0}+p \rho_{0}\right)+2 p \rho_{0}\left(1+\eta_{0} \eta_{1}\right)}{2 \eta_{0}\left(1-\rho_{0}\right)^{2}\left(1-\rho_{0}+p \rho_{0}\right)} .
$$

By using these values in (4.19), we invert (4.9) to obtain the corresponding $\rho$-value, from which we finally obtain an approximation for $\lambda=s \rho$.

In Table 5 numerical results are given of the approximation (4.18) of Equation (4.3) for $p=0.0005$ and $p=0.0001$ and several values of $s$. These values of $p$ and $s$ satisfy (4.8). We see that the results become worse as $s$ increases. To explain this, we observe that in the table the ratio $\lambda / s$ approaches unity for larger values of $s$. In that case the approximation (4.18) is not valid. It holds when $\rho$ is strictly less than unity.

To see the effect of smaller values of $p$ with $s$ fixed we give in Table 6 the results with $s=100$ and $s=1000$, and $p=2^{-n}, n=10,11,12, \ldots, 20$. We see, as expected, that the relative errors become smaller as $p$ decreases. 
Table 5: Numerical results of the approximation (4.18) of Equation (4.3) for $p=0.0005$ and $p=0.0001$ and several values of $s$.

\begin{tabular}{r|cc|cc} 
& \multicolumn{2}{|c|}{$p=0.0005$} & \multicolumn{2}{c}{$p=0.0001$} \\
\hline$s$ & $\lambda$ & rel. error & $\lambda$ & rel. error \\
\hline 5 & $0.6485810^{0}$ & $4.6510^{-6}$ & $0.4519510^{0}$ & $5.1310^{-7}$ \\
10 & $2.8027910^{0}$ & $8.0210^{-6}$ & $2.2601210^{0}$ & $8.9010^{-7}$ \\
25 & $1.2263610^{1}$ & $1.5710^{-5}$ & $1.0880010^{1}$ & $1.6910^{-6}$ \\
50 & $3.1292010^{1}$ & $2.6210^{-5}$ & $2.8866110^{1}$ & $2.7210^{-5}$ \\
75 & $5.1876710^{1}$ & $3.5510^{-5}$ & $4.8615010^{1}$ & $3.5910^{-6}$ \\
100 & $7.3248610^{1}$ & $4.4010^{-5}$ & $6.9264710^{1}$ & $4.3810^{-6}$ \\
250 & $2.0828710^{2}$ & $8.8910^{-5}$ & $2.0103410^{2}$ & $8.3010^{-6}$ \\
500 & $4.4254710^{2}$ & $1.5410^{-4}$ & $4.3141110^{2}$ & $1.3610^{-5}$ \\
1000 & $9.2173010^{2}$ & $2.7210^{-4}$ & $9.0482910^{2}$ & $2.2410^{-5}$
\end{tabular}

Table 6: Numerical results of the approximation (4.15) for $s=100$ and $s=1000$, and $p=2^{-n}$ for $n=10,11, \ldots, 20$.

\begin{tabular}{r|cc|cc} 
& \multicolumn{2}{|c|}{$s=100$} & \multicolumn{2}{c}{$s=1000$} \\
\hline$n$ & $\lambda$ & rel. error & $\lambda$ & rel. error \\
\hline 10 & $7.51710^{1}$ & $1.2410^{-4}$ & $9.30010^{2}$ & $8.8010^{-4}$ \\
11 & $7.31810^{1}$ & $4.2510^{-5}$ & $9.21510^{2}$ & $2.6210^{-4}$ \\
12 & $7.13810^{1}$ & $1.5410^{-5}$ & $9.13810^{2}$ & $8.5710^{-5}$ \\
13 & $6.97210^{1}$ & $5.7710^{-6}$ & $9.06710^{2}$ & $3.0010^{-5}$ \\
14 & $6.81810^{1}$ & $2.2410^{-6}$ & $9.00310^{2}$ & $1.1010^{-5}$ \\
15 & $6.67410^{1}$ & $8.8710^{-7}$ & $8.94210^{2}$ & $4.1810^{-6}$ \\
16 & $6.53910^{1}$ & $3.5910^{-7}$ & $8.88510^{2}$ & $1.6410^{-6}$ \\
17 & $6.41210^{1}$ & $1.4810^{-7}$ & $8.83110^{2}$ & $6.5410^{-7}$ \\
18 & $6.29110^{1}$ & $6.1810^{-8}$ & $8.78010^{2}$ & $2.6710^{-7}$ \\
19 & $6.17710^{1}$ & $2.6110^{-8}$ & $8.73210^{2}$ & $1.1010^{-7}$ \\
20 & $6.06710^{1}$ & $1.1210^{-8}$ & $8.68510^{2}$ & $4.6310^{-8}$
\end{tabular}

\section{Using the uniform asymptotic representation of $Q(s, \lambda)$}

The expansions used for the incomplete gamma function $Q(s, \lambda)$ (or $\Gamma(s, \lambda)$ ), see (3.1), are valid when $\lambda$ is large with respect to $s$. The expansion in (A.6) is more powerful; it holds when $r=s / \lambda$ belongs to an interval $\left[0, r_{0}\right]$, where $r_{0}$ is a fixed number, $r_{0}<1$. Next we consider an approximation of $Q(s, \lambda)$ that is valid for large $s$, and which holds uniformly with respect to $\lambda \geq 0$.

Let

$$
\operatorname{erfc} z=\frac{2}{\sqrt{\pi}} \int_{z}^{\infty} e^{-t^{2}} d t
$$

denote the complementary error function. We use quantities $\rho$ and $\eta$ defined by the relation in (4.9) (see also Appendix C). Then we have

$$
Q(s, \lambda)=\frac{1}{2} \operatorname{erfc}(\eta \sqrt{s / 2})+R_{s}(\eta),
$$


where $R_{s}(\eta)$ has the asymptotic representation

$$
R_{s}(\eta)=\frac{e^{-\frac{1}{2} s \eta^{2}}}{\sqrt{2 \pi s}} S_{s}(\eta), \quad S_{s}(\eta) \sim \sum_{n=0}^{\infty} \frac{C_{n}(\eta)}{s^{n}}, \quad s \rightarrow \infty .
$$

This asymptotic expansion is valid for $\eta \in \mathbb{R}$, that is, for $\rho \geq 0$, or $\lambda \geq 0$. A few details on the coefficients $C_{n}(\eta)$ are given in Appendix D.

The inversion problem (1.2) is written in the form (for the function $\Gamma^{*}$ see (4.5))

$$
B(s, \lambda)^{-1}=1+\sqrt{2 \pi s} \Gamma^{*}(s) e^{\frac{1}{2} s \eta^{2}} Q(s, \lambda),
$$

or as

$$
e^{\frac{1}{2} s \eta^{2}} Q(s, \lambda)=\frac{q}{\sqrt{2 \pi s} \Gamma^{*}(s)}, \quad q=\frac{1-p}{p} .
$$

Next we use (5.2), in which the complementary error function is the main approximant. We try to find a number $\eta_{0}$ defined by the equation

$$
\frac{1}{2} e^{\frac{1}{2} s \eta_{0}^{2}} \operatorname{erfc}\left(\eta_{0} \sqrt{s / 2}\right)=\frac{q}{\sqrt{2 \pi s} \Gamma^{*}(s)} .
$$

The left-hand side is a function of one variable, and, hence, the inversion of this equation is simpler than that of (5.5).

Let $y=\eta_{0} \sqrt{s / 2}$. The inversion problem (5.6) then reads

$$
f(y)=\frac{1}{2} e^{y^{2}} \operatorname{erfc} y-\kappa=0, \quad \kappa=\frac{q}{\sqrt{2 \pi s} \Gamma^{*}(s)} .
$$

We have

$$
f^{\prime}(y)=y e^{y^{2}} \operatorname{erfc} y-\frac{1}{\sqrt{\pi}}=2 y(f(y)+\kappa)-\frac{1}{\sqrt{\pi}} .
$$

Equation (5.7) can easily be solved numerically, for example by using Newton's method. When we have computed $y$ and $\eta_{0}=y \sqrt{2 / s}$ we can compute a first approximation of $\rho$, say $\rho_{0}$, from (4.9) with $\eta$ replaced with $\eta_{0}$ and $\operatorname{sign}\left(\eta_{0}\right)=\operatorname{sign}\left(\rho_{0}-1\right)$. After finding $\rho_{0}$, we have a first approximation of $\lambda$, say $\lambda_{0}$, from $\lambda_{0}=s \rho_{0}$.

In Table 7 we give the results of this first approximation $\lambda_{0}$ for fixed $s=100$ and several values of $p$. We see a better performance for values of $p$ near 0 and 1 . In Table 8 we give the results for fixed $p=0.1$ and $p=0.01$ and several values of $s$. We see that larger values of $s$ do not give a better approximation.

\subsection{Bounds}

From (5.4) and (5.2) we obtain

$$
B(s, \lambda)^{-1} \sim \frac{\sqrt{s}}{2 \phi(\eta \sqrt{s})} \operatorname{erfc}(\eta \sqrt{s / 2}),
$$

with $\phi(x)=\frac{1}{\sqrt{2 \pi}} e^{-\frac{1}{2} x^{2}}$. Including more terms of $R_{s}(\eta)$ in (5.2) is expected to yield sharper approximations. The approximation (5.9) can be complemented by bounds derived in [10]:

$$
\begin{aligned}
B(s, \lambda)^{-1} & \leq \frac{\sqrt{s}}{2 \phi(\eta \sqrt{s})} \operatorname{erfc}(\eta \sqrt{s / 2})+\frac{2}{3}+\frac{\sqrt{s}}{\phi(\eta \sqrt{s})(12 s-1)} \\
B(s, \lambda)^{-1} & \geq \frac{\sqrt{s}}{2 \phi(\eta \sqrt{s})} \operatorname{erfc}(\eta \sqrt{s / 2})+\frac{2}{3}
\end{aligned}
$$


Table 7: Numerical results of the approximation based on the inversion of (5.6) for $s=100$ and several values of $p$.

\begin{tabular}{cccc}
$p$ & $\lambda_{0}$ & $B\left(s, \lambda_{0}\right)$ & rel.error \\
\hline $1.00010^{-4}$ & $6.92610^{1}$ & $1.0000410^{-4}$ & $3.6410^{-5}$ \\
$1.00010^{-1}$ & $1.04710^{2}$ & $1.0341110^{-1}$ & $3.4110^{-2}$ \\
$2.00010^{-1}$ & $1.23010^{2}$ & $2.1351210^{-1}$ & $6.7610^{-2}$ \\
$3.00010^{-1}$ & $1.46410^{2}$ & $3.2989710^{-1}$ & $9.9710^{-2}$ \\
$4.00010^{-1}$ & $1.80210^{2}$ & $4.5150110^{-1}$ & $1.2910^{-1}$ \\
$5.00010^{-1}$ & $2.34210^{2}$ & $5.7611210^{-1}$ & $1.5210^{-1}$ \\
$6.00010^{-1}$ & $3.31610^{2}$ & $6.9968010^{-1}$ & $1.6610^{-1}$ \\
$7.00010^{-1}$ & $5.40410^{2}$ & $8.1536710^{-1}$ & $1.6510^{-1}$ \\
$8.00010^{-1}$ & $1.14410^{3}$ & $9.1267510^{-1}$ & $1.4110^{-1}$ \\
$9.00010^{-1}$ & $4.53710^{3}$ & $9.7796510^{-1}$ & $8.6610^{-2}$ \\
$9.99010^{-1}$ & $4.99810^{7}$ & $9.9999810^{-1}$ & $9.9910^{-4}$
\end{tabular}

Table 8: Numerical results of the approximation based on the inversion of (5.6) for $p=0.1$ and $p=0.01$ and several values of $s$.

\begin{tabular}{r|cc|cc} 
& \multicolumn{2}{|c|}{$p=0.1$} & \multicolumn{2}{c}{$p=0.01$} \\
\hline$s$ & $\lambda_{0}$ & rel. error & $\lambda_{0}$ & rel. error \\
\hline 5 & $2.92910^{0}$ & $3.9910^{-2}$ & $1.36210^{0}$ & $4.4810^{-3}$ \\
10 & $7.59710^{0}$ & $3.7310^{-2}$ & $4.46410^{0}$ & $4.0510^{-3}$ \\
25 & $2.30110^{1}$ & $3.5310^{-2}$ & $1.61310^{1}$ & $3.7310^{-3}$ \\
50 & $4.98810^{1}$ & $3.4610^{-2}$ & $3.79110^{1}$ & $3.5810^{-3}$ \\
75 & $7.71810^{1}$ & $3.4310^{-2}$ & $6.07410^{1}$ & $3.5310^{-3}$ \\
100 & $1.04710^{2}$ & $3.4110^{-2}$ & $8.40810^{1}$ & $3.4910^{-3}$ \\
250 & $2.70910^{2}$ & $3.3810^{-2}$ & $2.28310^{2}$ & $3.4210^{-3}$ \\
500 & $5.49010^{2}$ & $3.3710^{-2}$ & $4.74110^{2}$ & $3.3910^{-3}$ \\
1000 & $1.10610^{3}$ & $3.3610^{-2}$ & $9.71310^{2}$ & $3.3710^{-3}$
\end{tabular}

These bounds, and hence (5.9), are particularly sharp for the case $\lambda<s(\eta<0)$. The accuracy deteriorates with $\eta$, but improves when $s$ is increasing. Examples for $s=10$ and $s=20$ are depicted in Figures 2 and 3, respectively. In the present study we refrain from using the bounds for inversion purposes, since the asymptotic inversion introduced in $\S 5$ already leads to highly accurate results in the case $\lambda<s$. Bounds similar to but sharper than (5.10) and (5.11) can be found in [10].

\subsection{Higher order approximation}

It is possible to obtain higher approximations, as in [15] for the asymptotic inversion of the equation $Q(s, \lambda)=c, 0<c<1$, for large values of $s$, by using the uniform asymptotic representation of $Q(s, \lambda)$ given in (5.2). Then the inversion problem (5.5) can be written as

$$
e^{\frac{1}{2} s \eta^{2}}\left[\frac{1}{2} \operatorname{erfc}(\eta \sqrt{s / 2})+R_{s}(\eta)\right]=\frac{q}{\sqrt{2 \pi s} \Gamma^{*}(s)}, \quad q=\frac{1-p}{p} .
$$




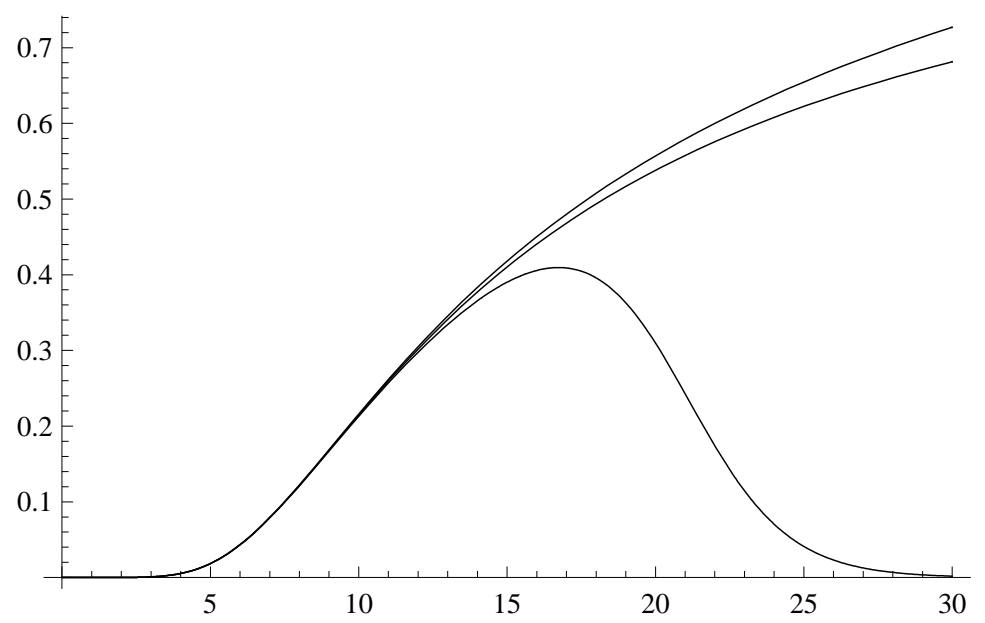

Figure 2: $B(s, \lambda)$ and lower and upper bounds for $s=10$ and $\lambda \in[0,30]$.

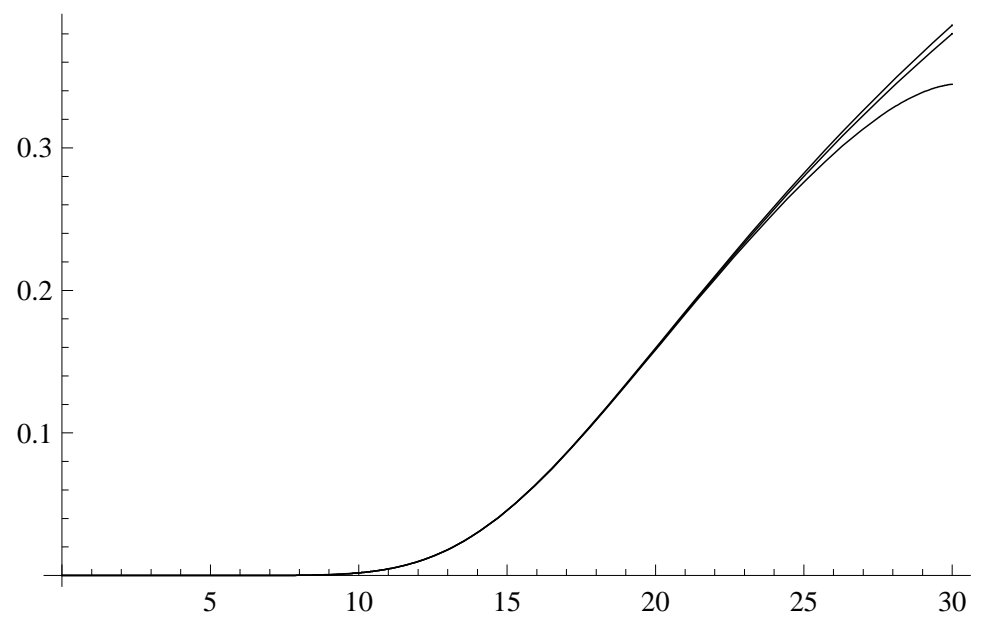

Figure 3: $B(s, \lambda)$ and lower and upper bounds for $s=20$ and $\lambda \in[0,30]$.

We can derive an asymptotic expansion of the solution $\eta$ of this equation in the form

$$
\eta \sim \eta_{0}+\frac{\eta_{1}}{s}+\frac{\eta_{2}}{s^{2}}+\ldots, \quad s \rightarrow \infty,
$$

where $\eta_{0}$ is the solution of (5.6) and the higher order coefficients $\eta_{j}, j \geq 1$ can be obtained by substituting the expansion (5.13) in to (5.12), after replacing $R_{s}(\eta)$ by the expansion given in (5.3). Details of this analysis are given in Appendix D. The first coefficient is found to be

$$
\eta_{1}=\frac{1}{\eta_{0}} \ln \left(1+\frac{\eta_{0} C_{0}\left(\eta_{0}\right)}{1-\eta_{0} q / \Gamma^{*}(s)}\right),
$$

where $C_{0}(\eta)$ is the first coefficient in the expansion in (5.3).

With $\eta \sim \eta_{0}+\eta_{1} / s$ the corresponding $\lambda$-value follows from solving (4.9) for $\rho$, from which we obtain an approximation $\lambda=\rho s$. The argument of the logarithm in (5.14) will become negative when $p \uparrow 1(q \downarrow 0)$, because $\eta_{0} q / \Gamma^{*}(s)$ will approach unity in that case. In Fig. 4 the upper curve corresponds to values $s$ and $p$ where the argument of the logarithm in (5.14) vanishes. 


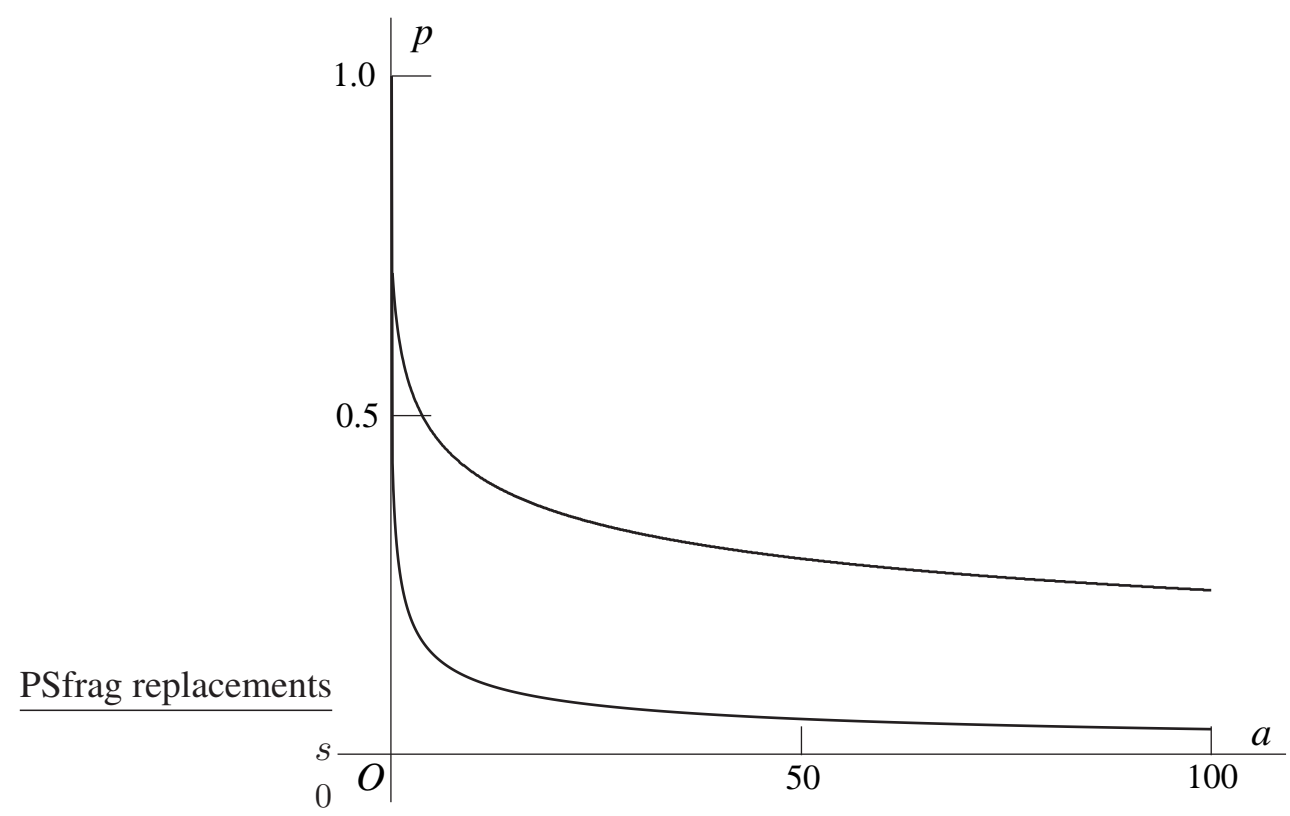

Figure 4: For values of $s$ and $p$ above the upper curve the argument of the logarithm in (5.14) is negative, and the expansion in (5.13) is useless in that case. The lower curve corresponds with values $s$ and $p$ where in (4.8) the equal sign holds.

In Table 9 we give the results of numerical computations. We take $p=0.1$ and $p=0.01$ and several values of $s$. We have used the term $\eta_{1}$ of (5.14) in the expansion (5.13). Comparing the results with those of Table 8 we see for $p=0.1$ an improvement for the smaller values of $s$. It appears that the argument of the logarithm in (5.14) becomes smaller as $s$ increases.

\section{Newton's method for inverting $B(s, \lambda)$}

Jagerman [9] uses Newton's method to derive an iteration that is well suited for determining the inverse $\lambda$ that solves the equation $B(s, \lambda)=p$. In the context of the present study, Newton's method can be applied for computing even better approximations of $\lambda$ through

$$
\lambda^{(n+1)}=\lambda^{(n)}-\frac{B\left(s, \lambda^{(n)}\right)-p}{\left(s / \lambda^{(n)}-1+B\left(s, \lambda^{(n)}\right)\right) B\left(s, \lambda^{(n)}\right)}, \quad n=0,1, \ldots,
$$

with $\lambda^{(0)}$ an asymptotic approximation.

For the asymptotic approximations given in $\S 3.1, \S 4$, and $\S 5.2$ we selected 30.000 random pairs $(p, s)$, with $s \in[5,1000]$ and $p$ in intervals which depend on the method.

For the asymptotic approximation (3.9) for $p \uparrow 1$ we used all coefficients $\lambda_{j}$ given in (4.9). We took $p \in(0.1,0.9999)$, and we found that the Newton process always converges to at least 10 digits accuracy in $\lambda$, with at most 4 iterations. For the asymptotic approximation (4.18) for small values of $p$ we used the coefficients $\eta_{j}$ given in (4.19)-(4.20). We took $p \in\left(3.0510^{-5}, 1.810^{-2}\right), p$ and $s$ such that $p \Gamma^{*}(s) \sqrt{2 \pi s} \leq 0.1$ (see Equation (4.8)), and found that the Newton process always converges to at least 10 digits accuracy in $\lambda$, with at most 3 iterations. Finally, for the approximation (5.13), with $\eta_{1}$ given in (5.14), we took $p \in\left(0.3010^{-5}, 0.5\right)$, and found that the Newton process always converges to at 
Table 9: Numerical results of the approximation based on the inversion of (5.12) for $p=$ 0.1 and $p=0.01$ and several values of $s$ by using (5.13), only with $\eta_{1}$ of (5.14).

\begin{tabular}{r|cc|cc} 
& \multicolumn{2}{|c|}{$p=0.1$} & \multicolumn{2}{c}{$p=0.01$} \\
\hline$s$ & $\lambda_{0}$ & rel. error & $\lambda_{0}$ & rel. error \\
\hline 5 & $2.88110^{0}$ & $3.5010^{-4}$ & $1.36110^{0}$ & $1.6710^{-5}$ \\
10 & $7.51010^{0}$ & $4.1510^{-4}$ & $4.46110^{0}$ & $4.0110^{-6}$ \\
25 & $2.28310^{1}$ & $6.3210^{-4}$ & $1.61210^{1}$ & $1.3610^{-6}$ \\
50 & $4.95510^{1}$ & $9.2410^{-4}$ & $3.79010^{1}$ & $1.3410^{-6}$ \\
75 & $7.67310^{1}$ & $1.1810^{-3}$ & $6.07310^{1}$ & $1.4910^{-6}$ \\
100 & $1.04110^{2}$ & $1.4310^{-3}$ & $8.40610^{1}$ & $1.6310^{-6}$ \\
250 & $2.69510^{2}$ & $2.8610^{-3}$ & $2.28310^{2}$ & $2.2010^{-6}$ \\
500 & $5.46410^{2}$ & $5.4210^{-3}$ & $4.74010^{2}$ & $2.7910^{-6}$ \\
1000 & $1.10010^{3}$ & $1.2110^{-2}$ & $9.71210^{2}$ & $3.6410^{-6}$
\end{tabular}

least 10 digits accuracy in $\lambda$, with at most 5 iterations. The small values of $p$ require only 2 iterations.

\section{The inversion of the Erlang $\mathrm{C}$ formula}

The Erlang $\mathrm{C}$ gives the steady-state probability of delay in the Erlang delay model or $M / M / s$ queue. It can be expressed in terms of the Erlang B formula as

$$
C(s, \lambda)^{-1}=\frac{\lambda}{s}+\left(1-\frac{\lambda}{s}\right) B(s, \lambda)^{-1}, \quad \lambda<s .
$$

Note that we now impose the condition $\lambda<s$. For more background on the Erlang $\mathrm{C}$ formula we refer to $[3,12,13,18]$. In this section we consider the inversion problem of $p=C(s, \lambda), p \in(0,1)$ for a given (possibly large) value of $s$.

Fig. 5 shows graphs of $C(s, \rho s)$ for $0 \leq \rho \leq 1$ and $s=1,3,5,10,25,50,100,1000$ (the far left curve is for $s=1$, the far right one for $s=1000$ ).

\subsection{Inversion of $C(s, \lambda)$ for $p \downarrow 0$}

We write the inversion problem in the form

$$
p^{-1}=\rho+(1-\rho)\left(1+\sqrt{2 \pi s} \Gamma^{*}(s) e^{\frac{1}{2} s \eta^{2}}-\sum_{n=0}^{\infty} \frac{g_{n}(\rho)}{s^{n}}\right), \quad \rho=\frac{\lambda}{s},
$$

where we have used (2.1), (2.2), (4.1), (4.5), (4.9), (4.10), and (B.6). We try to find the coefficients $\eta_{j}$ in the expansion

$$
\eta=\eta_{0}+\eta_{1} s^{-1}+\eta_{2} s^{-2}+\eta_{3} s^{-3} \ldots .
$$

To obtain the first coefficient $\eta_{0}$, we neglect in (7.2) the term $1-g_{0}(\rho)$, the remaining terms of the series, and the other two quantities $\rho$ (when $p$ is small, $\rho$ is small as well). Thus we consider (cf. (4.11))

$$
p^{-1}=\sqrt{2 \pi s} \Gamma^{*}(s) e^{\frac{1}{2} s \eta_{0}^{2}},
$$




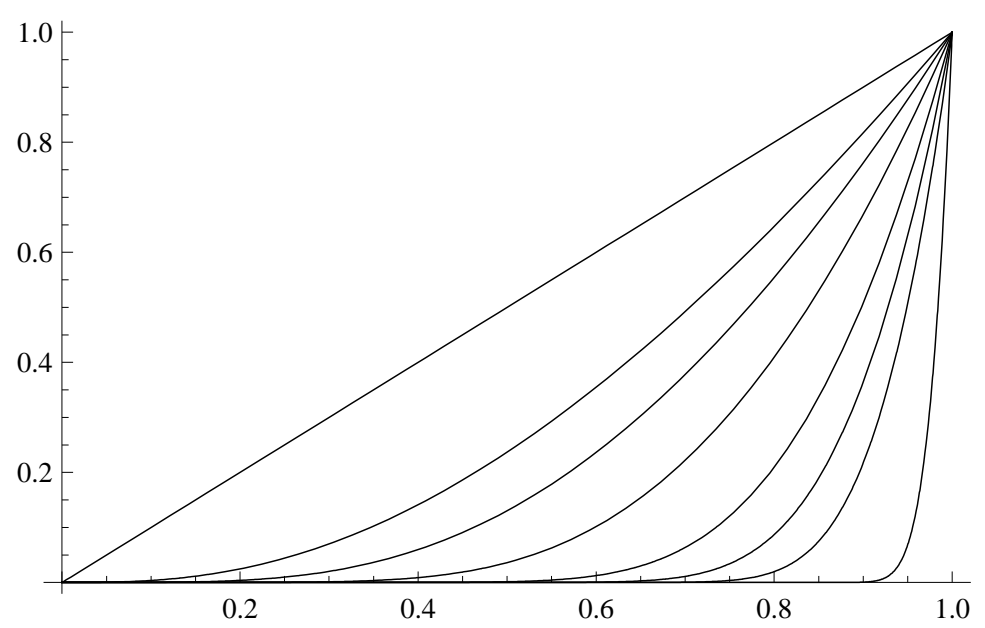

Figure 5: Graphs of $C(s, \rho s)$ for $0 \leq \rho \leq 1$ and $s=1,3,5,10,25,50,100,1000$ (the far left curve is for $s=1$, the far right one for $s=1000$.

where $p$ satisfies the same condition as for $p$ in (4.8). We obtain from (7.4) as a first approximation to $\eta$ the solution

$$
\eta_{0}=-\sqrt{-\frac{2}{s} \ln \left(p \sqrt{2 \pi s} \Gamma^{*}(s)\right)} .
$$

Proceeding as in $\S 4$ we eliminate $\sqrt{2 \pi s} \Gamma^{*}(s)$ by using (7.4), which gives for (7.1) the relation

$$
p^{-1}=\rho+(1-\rho)\left(1+\frac{1}{p} e^{\frac{1}{2} s\left(\eta^{2}-\eta_{0}^{2}\right)}-\sum_{n=0}^{\infty} \frac{g_{n}(\rho)}{s^{n}}\right) .
$$

Expanding $\rho$ as in (4.15) we find after series manipulations

$$
\begin{gathered}
\eta_{1}=-\frac{1}{\eta_{0}} \ln \left(1-\rho_{0}\right), \\
\eta_{2}=-\frac{\left.\eta_{1}^{2}\left(1-\rho_{0}\right)^{2}+2 \eta_{1} \rho_{0} \eta_{0}+2 p \rho_{0}\right)}{2 \eta_{0}\left(1-\rho_{0}\right)^{2}}
\end{gathered}
$$

and

$$
\eta_{3}=\frac{c_{3} \eta_{1}^{3}+c_{2} \eta_{1}^{2}+c_{1} \eta_{1}+c_{0}}{2 \eta_{0}^{2}\left(1-\rho_{0}\right)^{4}}
$$

where

$$
\begin{aligned}
& c_{0}=-p \eta_{0} \rho_{0}\left(-6 \rho_{0}+p \rho_{0}-2\right), \\
& c_{1}=2 \rho_{0}\left(p\left(1-\rho_{0}\right)^{2}+\eta_{0}^{2} \rho_{0}+p \rho_{0} \eta_{0}^{2}+p \eta_{0}^{2}\right), \\
& c_{2}=\eta_{0} \rho_{0}\left(2\left(1-\rho_{0}\right)^{2}+\eta_{0}^{2}\left(1+\rho_{0}\right)\right), \\
& c_{3}=\left(1-\rho_{0}\right)^{4} .
\end{aligned}
$$

When we have computed the values $\eta_{j}$ we use (7.3) for obtaining the approximation of $\eta$, from which the corresponding $\rho$ follows by inverting (4.9), which finally gives $\lambda=\rho s$.

In Table 10 numerical results are given of the approximation (7.3) of equation (7.2) for $p=0.0005$ and $p=0.0001$ and several values of $s$. We see that the results become worse 
Table 10: Numerical results of the approximation (7.3) of equation (7.2) for $p=0.0005$ and $p=0.0001$ and several values of $s$.

\begin{tabular}{r|cc|cc} 
& \multicolumn{2}{|c|}{$p=0.0005$} & \multicolumn{2}{c}{$p=0.0001$} \\
\hline$s$ & $\lambda$ & rel. error & $\lambda$ & rel. error \\
\hline 5 & $0.2889610^{0}$ & $2.4310^{-5}$ & $0.4428410^{0}$ & $3.8110^{-6}$ \\
10 & $2.6845710^{0}$ & $2.8310^{-4}$ & $2.1894110^{0}$ & $6.7010^{-5}$ \\
25 & $1.1683110^{1}$ & $2.4510^{-3}$ & $1.0474810^{1}$ & $6.9910^{-4}$ \\
50 & $2.9850810^{1}$ & $8.2210^{-3}$ & $2.7801510^{1}$ & $2.4510^{-3}$ \\
75 & $4.9586010^{1}$ & $1.5110^{-2}$ & $4.6888110^{1}$ & $4.5410^{-3}$ \\
100 & $7.0135110^{1}$ & $2.2510^{-2}$ & $6.6893810^{1}$ & $6.7610^{-3}$ \\
250 & $2.0068210^{2}$ & $7.0210^{-2}$ & $1.9516710^{2}$ & $2.0610^{-2}$ \\
500 & $4.2837910^{2}$ & $1.4910^{-1}$ & $4.2057610^{2}$ & $4.2910^{-2}$ \\
1000 & $8.9583610^{2}$ & $2.9110^{-1}$ & $8.8558510^{2}$ & $8.2910^{-2}$
\end{tabular}

as $s$ increases. To explain this, we observe that in the table the ratio $\lambda / s$ approaches unity for larger values of $s$. In that case the approximation (7.3) is not valid. To see the effect of smaller values of $p$ with $s$ fixed we give in Table 11 the results with $s=100$ and $s=1000$, and $p=2^{-n}, n=10,11,12, \ldots, 20$. We see, as expected, that the relative errors become smaller as $p$ decreases.

Table 11: Numerical results by using approximation (7.3) for $s=100$ and $s=1000$, and $p=2^{-n}$ for $n=10,11,12, \ldots, 20$.

\begin{tabular}{r|cc|cc} 
& \multicolumn{2}{|c|}{$s=100$} & \multicolumn{2}{c}{$s=1000$} \\
\hline$n$ & $\lambda$ & rel. error & $\lambda$ & rel. error \\
\hline 10 & $7.15910^{1}$ & $4.1810^{-2}$ & $8.97910^{2}$ & $5.3010^{-1}$ \\
11 & $7.00810^{1}$ & $2.2110^{-2}$ & $8.95710^{2}$ & $2.8510^{-1}$ \\
12 & $6.86410^{1}$ & $1.2610^{-2}$ & $8.91610^{2}$ & $1.6010^{-1}$ \\
13 & $6.72710^{1}$ & $7.7210^{-3}$ & $8.86910^{2}$ & $9.5210^{-2}$ \\
14 & $6.59710^{1}$ & $4.9510^{-3}$ & $8.82210^{2}$ & $6.0010^{-2}$ \\
15 & $6.47410^{1}$ & $3.3110^{-3}$ & $8.77610^{2}$ & $3.9610^{-2}$ \\
16 & $6.35610^{1}$ & $2.2910^{-3}$ & $8.73110^{2}$ & $2.7210^{-2}$ \\
17 & $6.24310^{1}$ & $1.6210^{-3}$ & $8.68710^{2}$ & $1.9310^{-2}$ \\
18 & $6.13510^{1}$ & $1.1810^{-3}$ & $8.64410^{2}$ & $1.4110^{-2}$ \\
19 & $6.03210^{1}$ & $8.7810^{-4}$ & $8.60310^{2}$ & $1.0510^{-2}$ \\
20 & $5.93210^{1}$ & $6.6310^{-4}$ & $8.56310^{2}$ & $8.0510^{-3}$
\end{tabular}

When we compare the results of the case $p \downarrow 0$ with the corresponding results for the Erlang B formula in $\S 4$, we observe that the present results are worse, even when we have one extra term in the expansion (7.3) (cf. (4.18)). An explanation might be that in the simplified Equation (7.4), for computing the first term $\eta_{0}$, more terms are neglected in (7.2) than in the corresponding case of $\S 4$ (cf. (4.3) and (4.11)). 


\subsection{Inversion of $C(s, \lambda)$ for $p \uparrow 1$}

We use in (7.1) representation (5.3) and obtain the inversion problem

$$
p^{-1}=\rho+(1-\rho)\left(1+\sqrt{2 \pi s} \Gamma^{*}(s) e^{\frac{1}{2} s \eta^{2}}\left[\frac{1}{2} \operatorname{erfc}(\eta \sqrt{s / 2})+R_{s}(\eta)\right]\right),
$$

which we solve for $\eta$ given in (4.9). When $p \uparrow 1$ we have for the solution $\lambda$ the estimate $\lambda \sim s$, that is, $\rho \sim 1 \sim-\eta$ (see (C.4)), and we consider for a first approximation to $\eta$ the reduced equation (compare with (5.6) and (5.7))

$$
\frac{1-p}{p \sqrt{\pi} \Gamma^{*}(s)}=-\eta \sqrt{s / 2} e^{\frac{1}{2} s \eta^{2}} \operatorname{erfc}(\eta \sqrt{s / 2}),
$$

the solution of which will be called $\eta_{0}$. This gives the first coefficient of the expansion (7.3).

To find higher coefficients $\eta_{j}$ we proceed as in $\S 5$ and Appendix D. We expand $\rho$ as in (4.15) and in this way we find the equivalent of (D.14), which reads for the present case

$$
\begin{gathered}
\sum_{n=0}^{\infty} C_{n}\left(\eta_{0}\right) s^{-n}+\frac{\varepsilon s}{1 !} \sum_{n=0}^{\infty} D_{n}^{(1)}\left(\eta_{0}\right) s^{-n}+\frac{(\varepsilon s)^{2}}{2 !} \sum_{n=0}^{\infty} D_{n}^{(2)}\left(\eta_{0}\right) s^{-n}+\ldots \\
\sim \frac{1-p}{\eta 0 p \Gamma^{*}(s)}\left[1-\frac{\eta_{0}}{\rho-1} e^{-\frac{1}{2} s\left(\eta^{2}-\eta_{0}^{2}\right)}\right] .
\end{gathered}
$$

The coefficients $D_{n}^{(k)}\left(\eta_{0}\right)$ are given in (D.15). Expanding in powers of $s^{-1}$, using (4.15), and comparing terms with powers $s^{0}$, we find

$$
\eta_{1}=\frac{1}{\eta_{0}} \ln \frac{\eta_{0}}{\rho_{0}-1}
$$

which, for small values of $\left|\eta_{0}\right|$, can be expanded as

$$
\eta_{1}=-\frac{1}{3}+\frac{1}{36} \eta_{0}+\frac{1}{1620} \eta_{0}^{2}-\frac{7}{6480} \eta_{0}^{3}+\ldots
$$

In Table 12 we give numerical results with $s=100$ and $s=1000$, and $p=1-2^{-n}$, $n=1,2, \ldots, 10$. We see a rather uniform relative error for these values of $p$ and $s$.

\subsection{Newton's method for inverting $C(s, \lambda)$}

For computing better approximations of $\lambda$ that solves the equation $C(s, \lambda)=p$, Newton's method result in the scheme

$$
\begin{aligned}
& \lambda^{(n+1)}=\lambda^{(n)}-\frac{C\left(s, \lambda^{(n)}\right)-p}{C^{\prime}\left(s, \lambda^{(n)}\right)}, \\
& C^{\prime}(s, \lambda)=\frac{d C(s, \lambda)}{d \lambda}=\frac{\left(\lambda+(s-\lambda)^{2}+\lambda(1-C(s, \lambda))\right) C(s, \lambda)}{\lambda(s-\lambda)},
\end{aligned}
$$

with $\lambda^{(0)}$ an asymptotic approximation.

For the asymptotic approximations given in $\S 7.1, \S 7.2$, we selected 30.000 random pairs $(p, s)$, with $s \in[5,1000]$ and $p$ in intervals which depend on the method. For the asymptotic approximation (7.3) for small values of $p$ we used the coefficients $\eta_{j}$ given in (7.7)(7.9). We took $p \in\left(3.0510^{-6}, 1.0110^{-2}\right)$, such that $p \Gamma^{*}(s) \sqrt{2 \pi s} \leq 0.1$ (see the remark after Equation (7.4)), and found that the Newton scheme always converges to at least 10 digits accuracy in $\lambda$, with at most 6 iterations. For the approximation $\eta \sim \eta_{0}+\eta_{1} / a$ of $\S 7.2$, with $\eta_{1}$ given in (7.14), we took $p \in(0.1,0.9999)$, and found that the Newton scheme always converges to at least 10 digits accuracy in $\lambda$, with at most 4 iterations. 
Table 12: Numerical results by using approximation (7.3) with $\eta_{1}$ given in (7.5) for $s=$ 100 and $s=1000$, and $p=1-2^{-n}$ for $n=1,2, \ldots, 10$.

\begin{tabular}{r|cc|cc} 
& \multicolumn{2}{|c|}{$s=100$} & \multicolumn{2}{c}{$s=1000$} \\
\hline$p$ & $\lambda$ & rel. error & $\lambda$ & rel. error \\
\hline $5.00010^{-1}$ & $9.47010^{1}$ & $3.1710^{-2}$ & $9.83810^{2}$ & $1.0310^{-2}$ \\
$7.50010^{-1}$ & $9.74810^{1}$ & $3.6310^{-2}$ & $9.92710^{2}$ & $1.1810^{-2}$ \\
$8.75010^{-1}$ & $9.86310^{1}$ & $3.8310^{-2}$ & $9.96410^{2}$ & $1.2410^{-2}$ \\
$9.37510^{-1}$ & $9.91610^{1}$ & $3.9210^{-2}$ & $9.98110^{2}$ & $1.2710^{-2}$ \\
$9.68810^{-1}$ & $9.94210^{1}$ & $3.9710^{-2}$ & $9.98910^{2}$ & $1.2910^{-2}$ \\
$9.84410^{-1}$ & $9.95410^{1}$ & $3.9910^{-2}$ & $9.99310^{2}$ & $1.3010^{-2}$ \\
$9.92210^{-1}$ & $9.96010^{1}$ & $4.0010^{-2}$ & $9.99510^{2}$ & $1.3010^{-2}$ \\
$9.96110^{-1}$ & $9.96410^{1}$ & $4.0110^{-2}$ & $9.99610^{2}$ & $1.3010^{-2}$ \\
$9.98010^{-1}$ & $9.96510^{1}$ & $4.0110^{-2}$ & $9.99610^{2}$ & $1.3010^{-2}$ \\
$9.99010^{-1}$ & $9.96610^{1}$ & $4.0110^{-2}$ & $9.99610^{2}$ & $1.3010^{-2}$
\end{tabular}

\section{A An alternative asymptotic expansion of $\Gamma(s, \lambda)$ for large $\lambda$}

The asymptotic expansion in (3.1) is of no use when $s$ becomes large as well. We give a different expansion in which the range of $s$ can be extended, say in the sense $s<\lambda-A \sqrt{\lambda}$, where $A$ is a fixed positive number and $\lambda$ is large.

We write

$$
\Gamma(s, \lambda)=\lambda^{s} \int_{1}^{\infty} t^{s-1} e^{-\lambda t} d t=\lambda^{s} e^{-\lambda} \int_{1}^{\infty} e^{-\lambda \psi(t)} \frac{d t}{t}
$$

where

$$
\psi(t)=t-r \ln t-1, \quad s=r \lambda .
$$

We write $f_{0}(t)=1$ and integrate by parts

$$
\Gamma(s, \lambda)=\lambda^{s} e^{-\lambda} \int_{1}^{\infty} \frac{f_{0}(t)}{t} e^{-\lambda \psi(t)} d t=-\lambda^{s-1} e^{-\lambda} \int_{1}^{\infty} \frac{f_{0}(t)}{t-r} d e^{-\lambda \psi(t)},
$$

which gives

$$
\Gamma(s, \lambda)=\lambda^{s-1} e^{-\lambda}\left[G_{0}(r)+\int_{1}^{\infty} e^{-\lambda \psi(t)} \frac{f_{1}(t)}{t} d t\right]
$$

where

$$
G_{0}(r)=\frac{1}{1-r}, \quad f_{1}(t)=t \frac{d}{d t} \frac{f_{0}(t)}{t-r}
$$

Continuing this we obtain

$$
\Gamma(s, \lambda)=\lambda^{s-1} e^{-\lambda}\left[\sum_{n=0}^{N-1} \frac{G_{n}(r)}{\lambda^{n}}+\frac{1}{\lambda^{N-1}} R_{N},\right], \quad N=0,1,2, \ldots,
$$

where

$$
f_{n}(t)=t \frac{d}{d t} \frac{f_{n-1}(t)}{t-r}, \quad G_{n}(r)=\frac{f_{n}(1)}{1-r}, \quad n=0,1,2, \ldots
$$


and

$$
R_{n}=\int_{1}^{\infty} e^{-\lambda \psi(t)} \frac{f_{n}(t)}{t} d t
$$

The expansion in (A.6) holds for large values of $\lambda$, with $r=s / \lambda$ satisfying $0 \leq r \leq$ $r_{0}<1$, where $r_{0}$ is fixed.

We have the recursion for the coefficients

$$
G_{n+1}(r)=\frac{r^{-n}}{r-1} \frac{d}{d r}\left[r^{n+1} G_{n}(r)\right], \quad n=1,2,3, \ldots,
$$

and the first few are given by

$$
\begin{aligned}
& G_{0}(r)=\frac{1}{1-r}, \quad G_{1}(r)=-\frac{1}{(1-r)^{3}}, \quad G_{2}(r)=\frac{2+r}{(1-r)^{5}}, \\
& G_{3}(r)=-\frac{6+8 r+r^{2}}{(1-r)^{7}}, \quad G_{4}(r)=\frac{24+58 r+22 r^{2}+r^{3}}{(1-r)^{9}} .
\end{aligned}
$$

The expansion in (A.6) is the same as the one given in [7, Eq. (19)], where it is derived for $\Gamma(s+1, \lambda)$. In Hwang's notation we have

$$
\tilde{f}_{0}(r)=G_{0}(r), \quad \tilde{f}_{j}(r)=(-1)^{j} r G_{j}(r), \quad j=1,2,3, \ldots .
$$

This relation follows from $\Gamma(s+1, \lambda)=s \Gamma(s, \lambda)+\lambda^{s} e^{-\lambda}$. The expansion in (A.6) follows also from [16, Eq. (4.1)], where it is derived with $s$ replaced with $-s$, with $s$ as the large parameter.

\section{B An alternative asymptotic expansion of $\gamma(s, \lambda)$ for large $s$}

The convergent expansion in (4.2) for the incomplete gamma function $\gamma(s, \lambda)$ has an asymptotic property for large values of $s$. When $s$ and $\lambda$ are of the same order this property is lost. We derive an asymptotic expansion for $\gamma(s, \lambda)$ that holds for large $s$ and $0 \leq \lambda \leq s(1-\delta)$, where $\delta$ is a fixed positive number, $\delta<1$. This expansion is of the same nature as the expansion for $\Gamma(s, \lambda)$ given in Appendix A, which holds for large $\lambda$ and $0 \leq s \leq \lambda(1-\delta)$.

We start with the integral representation

$$
\gamma(s, \lambda)=\int_{0}^{\lambda} t^{s-1} e^{-t} d t, \quad \Re s>0,
$$

and write it in the form

$$
e^{\lambda} \lambda^{-s} \gamma(s, \lambda)=\int_{0}^{1} e^{s \psi(t)} f_{0}(t) d t
$$

where

$$
f_{0}(t)=\frac{1}{1-t}, \quad \psi(t)=\rho t+\ln (1-t), \quad \rho=\frac{\lambda}{s} .
$$

We assume that $\rho \in(0,1)$. In that case, $\psi^{\prime}(t) \neq 0$ on $(0,1)$.

Integrating by parts gives (observe that $\psi(0)=0$ and $\psi^{\prime}(0)=\rho-1$ )

$$
e^{\lambda} \lambda^{-s} \gamma(s, \lambda)=\frac{1}{s} \int_{0}^{1} f_{0}(t) \frac{d e^{s \psi(t)}}{\psi^{\prime}(t)}=\frac{1}{s} \frac{f_{0}(0)}{1-\rho}+\frac{1}{s} \int_{0}^{1} e^{s \psi(t)} f_{1}(t) d t
$$


where

$$
f_{1}(t)=-\frac{d}{d t} \frac{f_{0}(t)}{\psi^{\prime}(t)}
$$

Repeating these steps gives the expansion

$$
e^{\lambda} \lambda^{-s} \gamma(s, \lambda) \sim \frac{1}{s} \sum_{n=0}^{\infty} \frac{g_{n}(\rho)}{s^{n}}, \quad g_{n}(\rho)=\frac{f_{n}(0)}{1-\rho},
$$

which holds as $s \rightarrow \infty$, uniformly for fixed $\rho \in(0,1)$, and where

$$
f_{n+1}(t)=-\frac{d}{d t} \frac{f_{n}(t)}{\psi^{\prime}(t)}, \quad n=0,1,2, \ldots,
$$

with $f_{0}(t)$ given in (B.3).

For $g_{n}(\rho)$ we have the recursion

$$
g_{n+1}(\rho)=-\frac{\rho}{1-\rho} \frac{d}{d \rho} g_{n}(\rho), \quad n=0,1,2, \ldots,
$$

and the first few are

$$
\begin{aligned}
& g_{0}(\rho)=\frac{1}{1-\rho}, \quad g_{1}(\rho)=-\frac{\rho}{(1-\rho)^{3}}, \quad g_{2}(\rho)=\frac{\rho(2 \rho+1)}{(1-\rho)^{5}} \\
& g_{3}(\rho)=-\frac{\rho\left(6 \rho^{2}+8 \rho+1\right)}{(1-\rho)^{7}}, \quad g_{4}(\rho)=\frac{\rho\left(24 \rho^{3}+58 \rho^{2}+22 \rho+1\right)}{(1-\rho)^{9}} .
\end{aligned}
$$

The relation with the coefficients $G_{n}(r)$ given in Appendix 2 for $\Gamma(s, \lambda)$ reads

$$
\rho^{n+1} g_{n}(\rho)=(-1)^{n} G_{n}(1 / \rho), \quad n=0,1,2, \ldots .
$$

\section{On the inversion of Equation (4.9)}

We recall the relation

$$
\frac{1}{2} \eta^{2}=\rho-1-\ln \rho, \quad \rho>0, \quad \operatorname{sign}(\eta)=\operatorname{sign}(\rho-1) .
$$

To invert this equation, we can use the Lambert $W$ function, the solution $W(x)$ of the equation $W e^{W}=x$. For example, we can write the equation in the form (using $y=\frac{1}{2} \eta^{2}$ )

$$
-\rho e^{-\rho}=-e^{-y-1} \quad \Rightarrow \quad \rho=e^{-W(-\exp (-y-1))-y-1},
$$

which is given by Maple, although $\rho=-W(-\exp (-y-1))$ can also be viewed as a formal solution. The problem is the multi-valuedness of this function, and we like to view the solution $\rho(\eta)$ of (C.1) as one analytic function on $\mathbb{R}$ (and in a certain domain of the complex plane, which is not our concern at this moment).

It is not difficult to compute $\rho(\eta)$, for example by using Newton's method, and it is convenient to have reliable starting values for this method. Let $\eta \neq 0$ and let $\rho^{(0)} \neq 1$ be a starting value. Then we can compute better approximations of $\rho$ through

$$
\rho^{(n+1)}=\rho^{(n)}-\frac{\rho^{(n)}-1-\ln \rho^{(n)}-\frac{1}{2} \eta^{2}}{1-1 / \rho^{(n)}}=\rho^{(n)} \frac{\ln \rho^{(n)}+\frac{1}{2} \eta^{2}}{\rho^{(n)}-1}, \quad n=0,1, \ldots
$$


For obtaining a starting value for small values of $|\eta|$, we can use the expansion

$$
\rho=1+\eta+\frac{1}{3} \eta^{2}+\frac{1}{36} \eta^{3}-\frac{1}{270} \eta^{4}+\frac{1}{4320} \eta^{5}+\mathcal{O}\left(\eta^{6}\right) .
$$

The complete expansion converges for $|\eta|<2 \sqrt{\pi}$ (as explained for a corresponding inversion in [17, pp. 284-286]).

When $\eta<0$ we have the convergent expansion

$$
\rho=\sum_{k=1}^{\infty} \frac{k^{k-1} \delta^{k}}{k !}, \quad \delta=e^{-1-\frac{1}{2} \eta^{2}} .
$$

This expansion converges very fast when $\eta$ is not close to zero, and it follows from applying the Lagrange inversion formula (see [4, pp. 22-23].

For $\eta \rightarrow+\infty$ we can use

$$
\rho=\tau+\sigma+\sum_{k=1}^{\infty} \frac{c_{k}}{\tau^{k}}, \quad \tau=1+\frac{1}{2} \eta^{2}, \quad \sigma=\ln \tau,
$$

where

$$
\begin{aligned}
& c_{1}=\sigma, \quad c_{2}=\sigma-\frac{1}{2} \sigma^{2}, \quad c_{3}=\sigma-\frac{3}{2} \sigma^{2}+\frac{1}{3} \sigma^{3}, \\
& c_{4}=\sigma-3 \sigma^{2}+\frac{11}{6} \sigma^{3}-\frac{1}{4} \sigma^{4} .
\end{aligned}
$$

This expansion follows from a similar analysis as given in [4, §2.5].

\section{Details on the uniform expansion and its asymptotic inversion}

The first few coefficients of the expansion in (5.3) are (see [14])

$$
\begin{aligned}
C_{0}(\eta) & =\frac{1}{\rho-1}-\frac{1}{\eta} & C_{0}(0) & =-\frac{1}{3} \\
C_{1}(\eta) & =\frac{1}{\eta^{3}}-\frac{1}{(\rho-1)^{3}}-\frac{1}{(\rho-1)^{2}}-\frac{1}{12(\rho-1)}, & C_{1}(0) & =-\frac{1}{540}
\end{aligned}
$$

and the higher coefficients an be obtained from the recurrence relation

$$
\eta C_{n}(\eta)=\frac{d}{d \eta} C_{n-1}(\eta)+\frac{\eta}{\rho-1} \gamma_{n}, \quad n \geq 1,
$$

where the numbers $\gamma_{n}$ appear in the well-known asymptotic expansion of the Euler gamma function. That is, we use (see also (4.5)) the asymptotic expansion

$$
\Gamma^{*}(s) \sim \sum_{n=0}^{\infty}(-1)^{n} \gamma_{n} s^{-n}, \quad s \rightarrow \infty
$$

where

$$
\gamma_{0}=1, \quad \gamma_{1}=-\frac{1}{12}, \quad \gamma_{2}=\frac{1}{288}, \quad \gamma_{3}=\frac{139}{51840}, \quad \gamma_{4}=-\frac{571}{2488320} .
$$

Next we give the analysis of the asymptotic inversion of the uniform expansion. We consider (5.12) and substitute $\eta=\eta_{0}+\varepsilon$, where $\eta_{0}$ is the solution of (5.6). We assume that $\varepsilon$ is small and expand in Taylor series. In this way we obtain

$$
\frac{1}{2} \sum_{k=1}^{\infty} \frac{\varepsilon^{k}}{k !} \frac{d^{k}}{d \eta^{k}} \operatorname{erfc}(\eta \sqrt{s / 2})+\sum_{k=0}^{\infty} \frac{\varepsilon^{k}}{k !} \frac{d^{k}}{d \eta^{k}} R_{s}(\eta)=\frac{q\left(e^{-\frac{1}{2} s \eta^{2}}-e^{-\frac{1}{2} s \eta_{0}^{2}}\right)}{\sqrt{2 \pi s} \Gamma^{*}(s)},
$$


where the derivatives are evaluated at $\eta_{0}$.

We replace the derivatives of $R_{s}(\eta)$ by derivatives of the expansion in (5.3) (from asymptotic analysis it follows that the expansion may be differentiated). In this way,

$$
\frac{d^{k}}{d \eta^{k}} R_{s}(\eta) \sim s^{k} \frac{e^{-\frac{1}{2} s \eta^{2}}}{\sqrt{2 \pi s}} \sum_{n=0}^{\infty} \frac{C_{n}^{(k)}(\eta)}{s^{n}},
$$

where

$$
C_{n}^{(0)}(\eta)=C_{n}(\eta), \quad n \geq 0, \quad C_{0}^{(k)}(\eta)=-\eta C_{0}^{(k-1)}(\eta), \quad k \geq 1,
$$

and

$$
C_{n}^{(k)}(\eta)=-\eta C_{n}^{(k-1)}(\eta)+\frac{d}{d \eta} C_{n-1}^{(k-1)}(\eta), \quad k, n \geq 1 .
$$

Observe that $C_{n}^{(k)}(\eta)$ is not the $k$ th derivative of $C_{n}(\eta)$. The relation (D.8) follows easily from the relations in (5.3).

The derivatives of the complementary error function in (D.5) can be replaced by derivatives of the exponential function, see (5.1), and we can use Hermite polynomials. We have $[17$, p. 145]

$$
H_{n}(z)=(-1)^{n} e^{z^{2}} \frac{d^{n}}{d z^{n}} e^{-z^{2}}, \quad n=0,1,2, \ldots,
$$

and it follows that for $k \geq 1$

$$
\frac{1}{2} \frac{d^{k}}{d \eta^{k}} \operatorname{erfc}(\eta \sqrt{s / 2})=(-1)^{k}\left(\frac{1}{2} s\right)^{\frac{1}{2} k} \frac{e^{-\frac{1}{2} s \eta^{2}}}{\sqrt{\pi}} H_{k-1}(\eta \sqrt{s / 2}) .
$$

We wish to write this in the same form as the right-hand side of (D.6). This can be done by using the explicit representation of the Hermite polynomials. That is, we use [17, p. 153]

$$
H_{n}(z)=n ! \sum_{m=0}^{\lfloor n / 2\rfloor}(-1)^{m} \frac{(2 z)^{n-2 m}}{m !(n-2 m) !}
$$

This gives

$$
\frac{1}{2} \frac{d^{k}}{d \eta^{k}} \operatorname{erfc}(\eta \sqrt{s / 2})=s^{k} \frac{e^{-\frac{1}{2} s \eta^{2}}}{\sqrt{2 \pi s}} \sum_{n=0}^{\lfloor(k-1) / 2\rfloor} \frac{h_{n}^{(k)}(\eta)}{s^{n}}, \quad k \geq 1,
$$

where, for $k \geq 1$ and $n=0,1,2, \ldots,\lfloor(k-1) / 2\rfloor$,

$$
h_{n}^{(k)}(\eta)=(-1)^{k+n} 2^{-n} \eta^{k-1-2 n} \frac{(k-1) !}{n !(k-1-2 n) !} .
$$

Substituting (D.6) and (D.12) into (D.5), we obtain, after some manipulations, the asymptotic equality

$$
\begin{gathered}
\sum_{n=0}^{\infty} C_{n}(\eta) s^{-n}+\frac{\varepsilon s}{1 !} \sum_{n=0}^{\infty} D_{n}^{(1)}(\eta) s^{-n}+\frac{(\varepsilon s)^{2}}{2 !} \sum_{n=0}^{\infty} D_{n}^{(2)}(\eta) s^{-n}+\ldots \\
\sim \frac{q\left(e^{-\frac{1}{2} s \varepsilon^{2}-s \eta \varepsilon}-1\right)}{\Gamma^{*}(s)}
\end{gathered}
$$


with $\eta=\eta_{0}$ and where the coefficients $D_{n}^{(k)}(\eta)$ are given by

$$
D_{n}^{(k)}(\eta)=C_{n}^{(k)}(\eta)+h_{n}^{(k)}(\eta), \quad n \geq 0, \quad k \geq 1 .
$$

We assume an expansion of $\varepsilon$ of the form (cf. (5.13))

$$
\varepsilon \sim \frac{\eta_{1}}{s}+\frac{\eta_{2}}{s^{2}}+\ldots, \quad s \rightarrow \infty,
$$

and collect coefficients of equal powers of $s$. In this way we can find $\eta_{k}$. For $\eta_{1}$ we find the equation

$$
C_{0}\left(\eta_{0}\right)+\sum_{k=1}^{\infty} \frac{\eta_{1}^{k}}{k !} D_{0}^{(k)}\left(\eta_{0}\right)=\frac{q\left(e^{-\eta_{0} \eta_{1}}-1\right)}{\Gamma^{*}(s)} .
$$

From (D.7) and (D.13) we find

$$
C_{0}^{(k)}(\eta)=(-\eta)^{k} C_{0}(\eta), \quad h_{0}^{(k)}(\eta)=-(-\eta)^{k-1}, \quad k \geq 1 .
$$

This gives

$$
D_{0}^{(k)}(\eta)=-(-\eta)^{k-1}\left(1+\eta C_{0}(\eta)\right), \quad k \geq 1 .
$$

Evaluating the series in (D.17) we obtain

$$
\eta_{0} C_{0}\left(\eta_{0}\right)+\left(1+\eta_{0} C_{0}\left(\eta_{0}\right)\right)\left(e^{-\eta_{0} \eta_{1}}-1\right)=\eta_{0} \frac{q\left(e^{-\eta_{0} \eta_{1}}-1\right)}{\Gamma^{*}(s)} .
$$

Solving for $\eta_{1}$ gives the value used in (5.14). That is,

$$
\eta_{1}=\frac{1}{\eta_{0}} \ln \left(1+\frac{\eta_{0} C_{0}\left(\eta_{0}\right)}{1-\eta_{0} q / \Gamma^{*}(s)}\right) .
$$

\section{Acknowledgements}

The work of JvL was supported by a VENI grant from The Netherlands Organization for Scientific Research (NWO). NMT acknowledges financial support from Ministerio de Educación y Ciencia, project MTM2006-09050 and from the Gobierno of Navarra, Res. 07/05/2008.

\section{References}

[1] S. A. Berezner, A. E. Krzesinski, and P. G. Taylor. On the inverse of Erlang's function. J. Appl. Probab., 35(1):246-252, 1998.

[2] E. Brockmeyer, H. L. Halstrøm, and A. Jensen. The life and works of A. K. Erlang. Trans. Danish Acad. Tech. Sci., 1948(2):277, 1948.

[3] R. B. Cooper. Introduction to Queueing Theory. North-Holland Publishing Co., New York, second edition, 1981.

[4] N. G. de Bruijn. Asymptotic Methods in Analysis. Dover Publications Inc., New York, third edition, 1981.

[5] A. Gil, J. Segura, and N. M. Temme. Numerical methods for special functions. SIAM, Philadelphia, PA, 2007. 
[6] A. Harel. Bounds, optimization and approximation results for the Erlang delay and loss formulae. Unpublished manuscript.

[7] H. K. Hwang. Asymptotic estimates of elementary probability distributions. Stud. Appl. Math., 99(4):393-417, 1997.

[8] D. L. Jagerman. Some properties of the Erlang loss function. Bell System Tech. J., 53:525-551, 1974.

[9] D. L. Jagerman. Methods in traffic calculations. AT\&T Bell Labs. Tech. J., 63(7):1283-1310, 1984.

[10] A. J. E. M. Janssen, J. S. H. van Leeuwaarden, and B. Zwart. Gaussian expansions and bounds for the Poisson distribution with application to the Erlang B formula. To appear in Advances of Applied Probability.

[11] L. Kosten. Stochastic Theory of Service Systems. Pergamon Press, Oxford, 1973. International Series of Monographs in Pure and Applied Mathematics, Vol. 103.

[12] J. Riordan. Stochastic Service Systems. The SIAM Series in Applied Mathematics. John Wiley and Sons, Inc., New York-London, 1962.

[13] R. Syski. Introduction to Congestion Theory in Telephone Systems. Foreword by Sir Thomas Eades; Chapter 2 by N. H. G. Morris. Published for Automatic Telephone and Electric Company Limited by Oliver and Boyd, Edinburgh-London, 1960.

[14] N. M. Temme. The asymptotic expansion of the incomplete gamma functions. SIAM J. Math. Anal., 10(4):757-766, 1979.

[15] N. M. Temme. Asymptotic inversion of incomplete gamma functions. Math. Comp., 58(198):755-764, 1992.

[16] N. M. Temme. Computational aspects of incomplete gamma functions with large complex parameters. In Approximation and computation (West Lafayette, IN, 1993), volume 119 of Internat. Ser. Numer. Math., pages 551-562. Birkhäuser Boston, Boston, MA, 1994.

[17] N. M. Temme. Special functions: An Introduction to the Classical Functions of Mathematical Physics. A Wiley-Interscience Publication. John Wiley \& Sons Inc., New York, 1996.

[18] W. Whitt. The Erlang B and C formulas: problems and solutions. Unpublished manuscript. 